\title{
Renormalized diffractive parton densities
}

\author{
Konstantin Goulianos ${ }^{* \dagger}$ \\ The Rockefeller University, 1230 York Avenue, New York, NY 10023, USA \\ E-mail: dino@mail.rockefeller.edu
}

\begin{abstract}
Data on soft and hard diffraction from deep inelastic scattering and $p p / p \bar{p}$ collisions are interpreted in a phenomenological model in which cross sections are obtained from the underlying inclusive parton distribution function of the nucleon using QCD color factors and appropriate (re)normalization. Experimental results on factorization breakdown in single-gap, and restoration of factorization in multi-gap diffraction are presented and compared with predictions of the model. Expectations for diffractive production at the Large Hadron Collider are discussed.
\end{abstract}

DIFFRACTION 2006 - International Workshop on Diffraction in High-Energy Physics

September 5-10 2006

Adamantas, Milos island, Greece

\footnotetext{
* Speaker.

${ }^{\dagger}$ http://physics.rockefeller.edu/dino/my.html
} 


\section{Introduction}

Hadronic diffraction is characterized by one or more large rapidity gaps, defined as regions of rapidity ${ }^{1}$ devoid of particles. Rapidity gaps may occur in non-diffractive (ND) interactions by fluctuations in particle multiplicity. However, the probability for such occurrences is expected to be exponentially suppressed as a function of gap width, since at a given $\sqrt{s}$ the particle density $\rho_{N}=d N / d \eta$ is approximately constant versus $\eta$ and therefore the probability for no particles being produced at $(\eta, \Delta \eta)$ is by Poisson statistics $P_{0}(\eta, \Delta \eta)=e^{-\rho_{N} \Delta \eta}$ [1]. Diffractive rapidity gaps do not exhibit such a suppression. This aspect of diffraction is attributed to the lack of radiation in the diffractive exchange, which proceeds through a colorless quark / gluon construct with vacuum quantum numbers, historically referred to as Pomeron [2]. In this paper, we use the term Pomeron generically to denote a diffractive exchange, and describe it in terms of the underlying parton densities of the proton as a function of $Q^{2}$. Our phenomenological description is driven by regularities observed in results on soft and hard diffractive processes obtained in $p p / p^{-} p$ collisions in fixed target and collider experiments, and in ep collisions at HERA (see also review in Ref. [3] ).
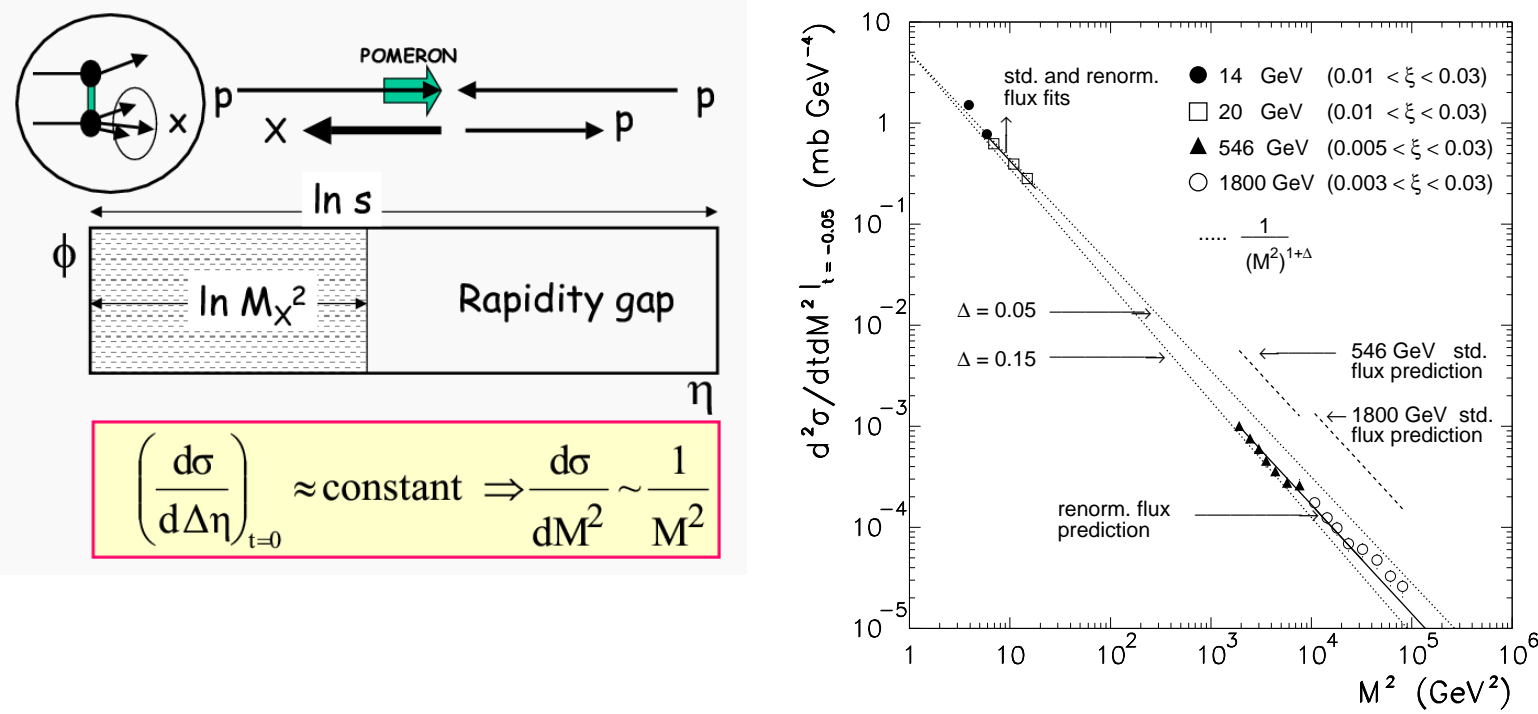

Figure 1: (left) Event topology for $p p$ single diffraction dissociation, $p p \rightarrow p X$ : lack of radiation in "vacuum exchange" leads to a cross section independent of $\Delta \eta$ and thereby to $1 / M^{2}$ behavior; (right) Cross sections $d^{2} \sigma_{s d} / d M^{2} d t$ for $p+p(\bar{p}) \rightarrow p(\bar{p})+X$ at $t=-0.05 \mathrm{GeV}^{2}$ and $\sqrt{s}=14,20,546$ and $1800 \mathrm{GeV}$ compared with the renormalized Pomeron flux prediction 肺 ( $\Delta$ is the Pomeron intercept, denoted by $\varepsilon$ through the rest of this report). At $\sqrt{s}=14$ and $20 \mathrm{GeV}$, the fits using the standard and renormalized fluxes coincide; at the higher energies, the standard flux prediction overestimates the cross sections by $\sim \mathscr{O}(10)$.

A clue to understanding diffraction in QCD is provided by the $d \sigma / d M^{2}$ behavior of the soft single diffractive cross section. As shown in Fig. 1 (left), due to absence of radiation in vacuum exchange, a $d \sigma / d M^{2} \sim 1 / M^{2}$ behavior is expected with no explicit $s$-dependence. This is observed in data in Fig. 1 (right). The deviation from exact $1 / M^{2}$ behavior holds the key to understanding diffractive cross sections in terms of the underlying parton densities of the diffracted nucleon.

\footnotetext{
${ }^{1}$ We use rapidity, $y=\frac{1}{2} \frac{E+p_{L}}{E-p_{L}}$, and pseudorapidity, $\eta=-\ln \tan \frac{\theta}{2}$, interchangeably, as they are numerically close in the kinematic region of interest in this discussion.
} 


\section{The data}

Scaling and factorization properties observed in data provide the input to our model of describing hadronic diffraction in terms of inclusive parton densities and QCD color factors. The foundations of the model are mainly results from the CDF experiment at the Tevatron $p^{-} p$ collider at Fermilab, and from the H1 and ZEUS experiments at the HERA ep collider at DESY. Reviews of these results have been presented at this conference [5, 6, 7].

Table 1 lists the soft diffractive processes studied by CDF in Run I. Measurement details and results can be found in the references provided.

Table 1: Soft diffractive processes studied by CDF
ND Non-Diffractive $\left(\sigma_{T}\right)$
$\bar{p}+p \rightarrow X^{\dagger}$
EL Elastic [9]
$\bar{p}+p \rightarrow \bar{p}+p$
SD Single Diffraction [10]
$\bar{p}+p \rightarrow \bar{p}+$ gap $+X$
DD Double Diffraction [11]
$\bar{p}+p \rightarrow X+$ gap $+Y$
DPE Double Pomeron Exchange [12]
$\bar{p}+p \rightarrow \bar{p}+$ gap $+X+$ gap $+p$
SDD Single plus Double Diffraction [13]
$\bar{p}+p \rightarrow \bar{p}+$ gap $+X+$ gap $+Y$
$\dagger \sigma_{T}$ is included since by the optical theorem it is related to $\operatorname{Im} f^{e l}(t=0)$

Hard diffractive processes studied by CDF include $J J$ (dijet), $W, b$-quark, and $J / \psi$ production. Results from Run I have been published in Phys. Rev. Letters (see review in Ref. [3]), and preliminary Run II results have been presented at conferences, including the present one [5].

The most interesting aspects of the results in connection with the QCD structure of the diffractive exchange are the breakdown of factorization and the restoration of factorization in events with multiple rapidity gaps. The following two paragraphs are based on excerpts from Ref. [5], and are provided here for convenience.

Breakdown of factorization. At $\sqrt{s}=1800 \mathrm{GeV}$, the $\mathrm{SD} / \mathrm{ND}$ ratios (gap fractions) for dijet, $W$, $b$-quark, and $J / \psi$ production, as well the ratio of $\mathrm{DD} / \mathrm{ND}$ dijet production, are all $\approx 1 \%$. This represents a suppression of $\sim \mathscr{O}(10)$ relative to predictions based on diffractive parton densities measured from Diffractive Deep Inelastic Scattering (DDIS) at HERA, indicating a breakdown of QCD factorization comparable to that observed in soft diffraction processes relative to Regge theory expectations (see section 11). However, factorization approximately holds among the above four hard diffractive processes at fixed $\sqrt{s}$, which suggests that the suppression is in the rapidity gap formation probability, as predicted by the generalized gap renormalization model (RENORM), which is the subject of the present paper (see also review in Ref. [3]).

Restoration of factorization in multi-gap diffraction. Another interesting CDF result is that ratios of two-gap to one-gap cross sections for both soft and hard processes obey factorization. This provides not only a clue to understanding diffraction in terms of a composite Pomeron, but also an experimental discovery tool for new physics using processes with multiple rapidity gaps. 


\section{Renormalized diffractive cross sections: soft diffraction}

Diffraction has traditionally been treated in Regge theory using factorization. This approach was successful at $\sqrt{s}$ energies below $\sim 50 \mathrm{GeV}$ [14], but as the available energies increased to $\sqrt{s}=1800 \mathrm{GeV}$ in Run I at the Tevatron, a suppression as large as $\sim \mathscr{O}(10)$ of the SD cross section was observed relative to the Regge theory based predictions [10]. This breakdown of factorization was traced to the energy dependence of $\sigma_{s d}^{t o t}(s) \sim s^{2 \varepsilon}$, which is faster than that of $\sigma^{t o t}(s) \sim s^{\varepsilon}$, so that at high $\sqrt{s}$ unitarity would have to be violated if factorization held. The $s$-dependence appears explicitly in the SD differential cross section:

$$
\text { Regge theory: } d \sigma_{s d}\left(s, M^{2}\right) / d M^{2} \sim \frac{s^{2 \varepsilon}}{\left(M^{2}\right)^{1+\varepsilon}} .
$$

As seen in Fig. 1 (right), contrary to the Regge theory based expectation of Eq. (3.1), the measured SD $M^{2}$-distribution does not show any $s$-dependence over a region of $s$ six orders of magnitude. Thus, it appears that factorization breaks down in such a way as to enforce $M^{2}$-scaling. This property is built into RENORM, in which the Regge theory Pomeron flux is renormalized to unity [15]. Below, we present a QCD basis for renormalization and its extension to central and multi-gap diffraction [16].

The form of the rise of total cross sections at high energies, $\sim s^{\varepsilon}$, which in Regge theory requires a Pomeron trajectory with intercept $\alpha(0)=1+\varepsilon$, is expected in a parton model approach, where cross sections are proportional to the number of available wee partons [17]. In terms of the rapidity region in which there is particle production ${ }^{2}, \Delta \eta^{\prime}$, the total $p p$ cross section is given by

$$
\sigma_{p p}^{t o t}=\sigma_{0} \cdot e^{\varepsilon \Delta \eta^{\prime}}
$$

Since from the optical theorem $\sigma_{t o t} \sim \operatorname{Imf}^{\mathrm{el}}(t=0)$, the full parton model amplitude takes the form

$$
\operatorname{Imf}^{\mathrm{el}}(t, \Delta \eta) \sim e^{\left(\varepsilon+\alpha^{\prime} t\right) \Delta \eta}
$$

where the term $\alpha^{\prime} t$ is a parameterization of the $t$-dependence of the amplitude. Based on this amplitude, the diffractive cross sections of table 1 are expected to have the forms

$$
\begin{aligned}
\frac{d^{2} \sigma_{s d}}{d t d \Delta \eta} & =N_{\text {gap }}^{-1}(s) \quad F_{p}(t)\left\{e^{\left[\varepsilon+\alpha^{\prime}(t)\right] \Delta \eta}\right\}^{2} \quad \kappa\left[\sigma_{0} e^{\varepsilon \Delta \eta^{\prime}}\right] \\
\frac{d^{3} \sigma_{d d}}{d t d \Delta \eta d \eta_{c}} & =N_{\text {gap }}^{-1}(s) \quad\left\{e^{\left[\varepsilon+\alpha^{\prime}(t)\right] \Delta \eta}\right\}^{2} \quad \kappa\left[\sigma_{0} e^{\varepsilon\left(\Sigma_{i} \Delta \eta_{i}^{\prime}\right)}\right] \\
\frac{d^{4} \sigma_{s d d}}{d t_{1} d t_{2} d \Delta \eta d \eta_{c}} & =N_{\text {gap }}^{-1}(s) \quad F_{p}(t) \Pi_{i}\left\{e^{\left[\varepsilon+\alpha^{\prime}\left(t_{i}\right)\right] \Delta \eta_{i}}\right\}^{2} \kappa^{2}\left[\sigma_{0} e^{\varepsilon\left(\Sigma_{i} \Delta \eta_{i}^{\prime}\right)}\right] \\
\frac{d^{4} \sigma_{d p e}}{d t_{1} d t_{2} d \Delta \eta d \eta_{c}^{\prime}} & =N_{\text {gap }}^{-1}(s) \underbrace{\prod_{i}\left\{F_{p}\left(t_{i}\right) e^{\left[\varepsilon+\alpha^{\prime}\left(t_{i}\right)\right] \Delta \eta_{i}}\right\}^{2}}_{\text {gap probability }} \kappa^{2} \underbrace{\left[\sigma_{0} e^{\varepsilon\left(\Delta \eta^{\prime}\right)}\right]}_{\sigma^{\text {tot }}\left(s^{\prime}\right)}
\end{aligned}
$$

where the (re)normalization factor $N_{g a p}(s)$ is the integral of the gap probability over all phase space in $\left(t_{i}, \Delta \eta_{i}, \eta_{c}, \eta_{c}^{\prime}\right)$, and the variables $\eta_{c}$ and $\eta_{c}^{\prime}$ represent the center of the "floating" (not adjacent

${ }^{2}$ We take $p_{T}=1 \mathrm{GeV}$ so that $\Delta y^{\prime}=\Delta \eta^{\prime}$. 
to a nucleon) rapidity gap in DD or SDD and floating diffractive cluster in DPE, respectively. In each case, the independent variables are the ones on the left hand side of the equation, but for pedagogical reason we use on the right side the additional variables $\Delta \eta_{i}^{\prime}$, which could be be expressed in terms of $\ln s$ and the variables on the left.

The expressions in Eq. (3.4) are built from the following components:

- the reduced energy cross section, $\sigma_{p p / p \bar{p}}^{\text {tot }}\left(s^{\prime}\right)$, which is the $p p / p^{-} p$ cross section at the reduced collision energy, which is defined by the equation $\ln \left(s^{\prime} / s_{0}\right)=\sum_{i} \Delta y_{i}^{\prime}$;

- the color factors $\kappa$, one for each gap, required to select color neutral exchanges with vacuum quantum numbers to ensure diffractive rapidity gap formation;

- the gap probability, which is given by the amplitude squared of the elastic scattering between a diffractively dissociated and a surviving proton, in which case it contains the proton form factor, $F_{p}(t)$, or between two diffractively dissociated protons;

- the normalization factor $N_{g a p}^{-1}$, which is the inverse of the integral of the gap probability over all phase space.

A remarkable property of the expressions in Eq. (3.4) is that they factorize into two terms, one which depends on the sum of the rapidity regions in which there is particle production, and another which depends on the sum of the widths of the rapidity gaps. This is rendered possible by the exponential dependence on $\Delta \eta$ of the elastic amplitude, which allows non-contiguous regions in rapidity to be added in the exponent. A consequence of this type of factorization is that the (re)normalization factor is the same and $\sim s^{2 \varepsilon}$ in all cases, ensuring $M^{2}$-scaling and universality of the suppression factor across single, central, and multi-gap diffraction.

The parameters $\varepsilon$ and $\kappa$. Experimentally, these parameters have been measured to be [18, 4$]$

$$
\begin{aligned}
& \varepsilon \equiv \alpha_{\mathbb{P P}}(0)-1=0.104 \pm 0.002 \pm 0.01 \text { (syst), and } \\
& \kappa \equiv \frac{g_{\mathbb{P I P I P}}}{\beta_{\mathbb{P} P}}=0.17 \pm 0.02 \text { (syst) }
\end{aligned}
$$

where the systematic uncertainty assigned to $\varepsilon$ is an estimate based on considering results from fits made to cross section data by various authors. Measurements of parton densities at HERA indicate that partonic structure in the nucleon is expressed down to the hadron mass scale of $Q^{2} \approx 1 \mathrm{GeV}^{2}$. This is seen in Fig. 2 (left), where the parameter $\lambda\left(Q^{2}\right)$ of $F_{2}\left(x, Q^{2}\right) \sim x^{-\lambda\left(Q^{2}\right)}$ decreases linearly with $\ln Q^{2}$ down to $Q^{2} \approx 1 \mathrm{GeV}^{2}$, flattening out and becoming consistent with the soft Pomeron intercept only below $Q^{2}=1 \mathrm{GeV}^{2}$. We therefore assume partonic structure in soft diffractive exchanges at the hadron mass scale, and proceed with a "toy estimate" of the parameters $\varepsilon$ and $\kappa$ from the nucleon PDF, using the PDF at $Q^{2}=1 \mathrm{GeV}^{2}$ shown in Fig. 目 (right) obtained from the CTEQ5L parameterization. 

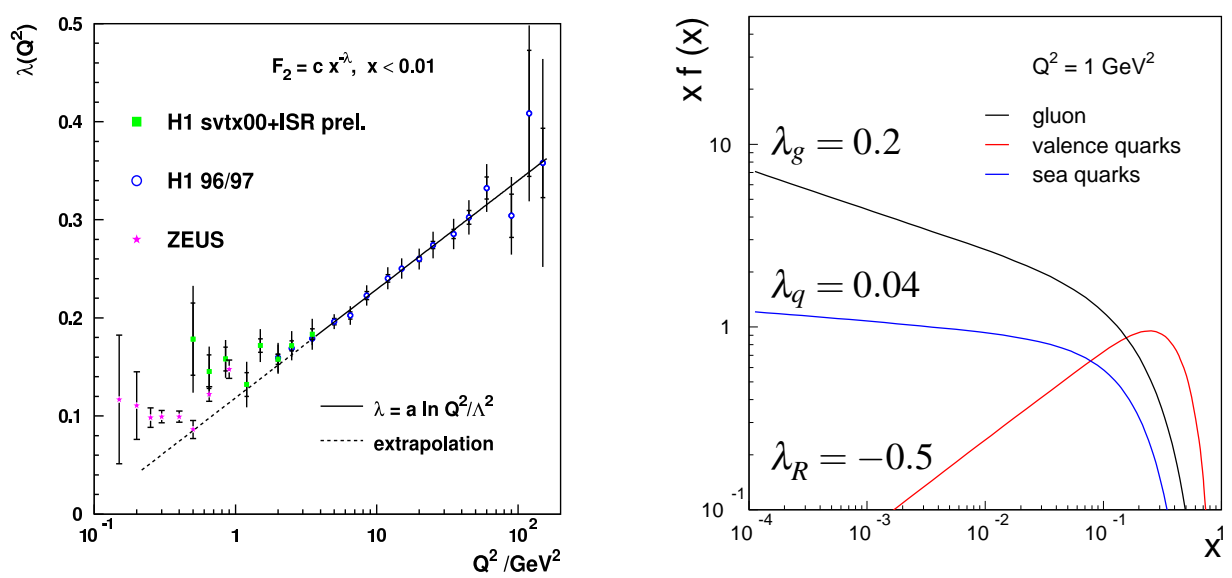

Figure 2: (left) The parameter $\lambda\left(Q^{2}\right)$ versus $Q^{2}$ of a fit to the structure function $F_{2}\left(x, Q^{2}\right) \sim x^{-\lambda\left(Q^{2}\right)}$ in DIS at HERA[[19]; (right) CTEQ5L nucleon parton distribution functions for $Q^{2}=1 \mathrm{GeV}^{2}$.

The region of interest to diffraction, $x \leq 0.1$, is dominated by sea gluons and quarks. In this region, a fit of the form $x f(x) \sim x^{-\lambda}$, Fig. 2 (right), yields $\lambda_{g} \approx 0.2$ and $\lambda_{q} \approx 0.04$ with relative weights $w_{g} \approx 0.75$ and $w_{q} \approx 0.25^{3}$. Noting that the number of wee partons grows as $\int_{1 / s}^{1} f(x) d x \sim s^{\lambda}$, the Pomeron intercept may be obtained from the parameters $\lambda_{g}$ and $\lambda_{q}$ appropriately weighted by the gluon and quark color factors

$$
c_{g}=\frac{1}{N_{c}^{2}-1}, \quad c_{q}=\frac{1}{N_{c}} .
$$

Weighting places $\varepsilon$ in the range $\lambda_{q}<\varepsilon<\lambda_{g}$, or $0.04<\varepsilon<0.2$, which covers the experimental value of $\varepsilon=0.104$. A precise determination is not attempted, as it would require averaging over the $Q^{2}$ range of all the particles produced in the collision and proper accounting of the uncertainties in the nucleon PDF in this low $Q^{2}$ region.

The parameter $\kappa$ is obtained from the gluon and quark color factors and weights:

$$
\kappa \approx c_{g} w_{g}+c_{q} w_{q}=0.182
$$

This RENORM prediction is in remarkably good agreement with $\kappa_{\text {exp }}=0.17 \pm 0.02$.

\section{Renormalized diffractive cross sections: hard diffraction}

Hard diffraction processes are defined as those in which there is a hard partonic scattering in addition to the diffractive rapidity gap signature. Events may have forward, central, or multiple rapidity gaps in topologies similar to those listed in table 1 for soft ${ }^{-} p p$ collisions at the Tevatron, with the hard scattering products appearing within the region(s) of rapidity where there is particle production.

\footnotetext{
${ }^{3}$ For valence quarks, $\lambda_{q} \equiv \lambda_{R} \approx-0.5$; this is relevant for Reggeon exchange, which is not being considered here, as its contribution is relatively small.
} 
Hard diffraction data. $\mathrm{CDF}$ has measured SD/ND ratios for $W$, dijet, $b$-quark and $J / \psi$ production, and also diffractive structure functions extracted from SD and DPE dijet production [5]. HERA has reported DPFs extracted from inclusivs DDIS, as well as from exclusive channels [6, 7].

The following interesting characteristics have been observed:

- all SD/ND ratios measured by $\mathrm{CDF}$ at $\sqrt{s}=1800 \mathrm{GeV}$ are approximately equal, pointing to a flavor independent rapidity gap formation probability;

- the dijet SD/ND ratio measured by CDF varies as $\sim x_{B j}^{-0.45}$, contrary to results from deep inelastic scattering at HERA, where a constant ratio of DDIS/DIS is observed [19, 6, 7];

- the SD structure function extracted from dijet production at CDF is suppressed by $\sim \mathscr{O}(10)$ relative to expectations from diffractive PDFs measured in DDIS at HERA;

- the Pomeron intercept measured in DDIS at HERA increases with $Q^{2}$ and is on average larger than the soft Pomeron intercept, but approximately a factor of $\sim 2$ smaller than the intercept obtained from inclusive DIS.

Diffractive parton densities. Diffractive parton densities with good statistics have been obtained from DDIS at HERA and diffractive dijet production at the Tevatron:

$$
\begin{aligned}
\text { HERA: } & \gamma^{*}+p \rightarrow p+j e t+X \\
\text { Tevatron: } & \bar{p}+p \rightarrow \bar{p}+\operatorname{dijet}+X
\end{aligned}
$$

The production process may involve several color "emissions" from the surviving proton, collectively comprising a color singlet with vacuum quantum numbers. Two of the emissions are of special importance: one at $x=x_{B j}$ from the proton's PDF at scale $Q^{2}$, which is responsible for the hard scattering, and another at $x=\xi$ (fractional momentum loss of the diffracted nucleon) from the PDF at scale $Q^{2} \approx 1 \mathrm{GeV}^{2}$, which neutralizes the exchanged color and forms the rapidity gap.

At HERA, at small $\xi$ where the proton PDF exhibits power law behavior at both soft and hard scales, the diffractive structure function takes the form ${ }^{4}$

$$
F_{2}^{D(3)}\left(\xi, x, Q^{2}\right)=\frac{A_{\text {norm }}}{\xi^{1+\varepsilon_{q}}} \cdot c_{q} \cdot F_{2}\left(x, Q^{2}\right)
$$

where $A_{\text {norm }}$ is a normalization factor, $\varepsilon_{q}$ is the exponent of the soft quark structure function, which from Fig. 2 (right) is given by $\varepsilon_{q}=\lambda_{q}\left(Q^{2}=1\right)=0.04$, and $c_{q}=1 / 3$ is the quark color factor.

\footnotetext{
${ }^{4}$ For simplicity, we do not consider the $t$ dependence in this discussion. This has little affect on our conclusions, as diffractive cross sections are concentrated at small $t$.
} 


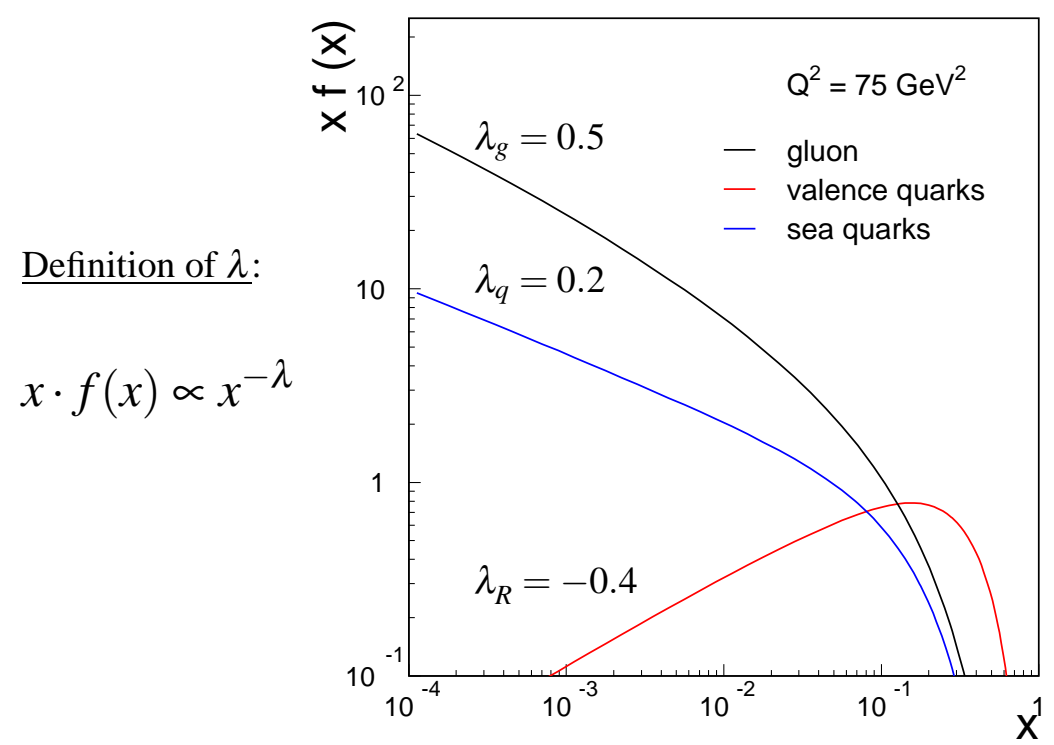

Figure 3: CTEQ5L nucleon parton distribution functions for $Q^{2}=75 \mathrm{GeV}^{2}$. The parameters $\lambda_{g, q, R}$ are the slopes of the gluon, sea quark, and valence quark distribution (' $R$ ' stands for Reggeon) in the region of $x<0.1$, where the power law behavior holds.

At high $Q^{2}$, where factorization is expected to hold [15, 20], $A_{\text {norm }}$ is the normalization factor of the soft PDF, which is a constant, and $F_{2}$ can be expressed as a power law, resulting in

$$
F_{2}^{D(3)}\left(\xi, x, Q^{2}\right)=\frac{A_{\text {norm }}}{\xi^{1+\varepsilon_{q}}} \cdot \frac{1}{3} \cdot \frac{C\left(Q^{2}\right)}{(\beta \xi)^{\lambda\left(Q^{2}\right)}}=\frac{A_{\text {norm }}}{\xi^{1+\varepsilon_{q}+\lambda\left(Q^{2}\right)}} \cdot \frac{1}{3} \cdot \frac{C\left(Q^{2}\right)}{\beta^{\lambda\left(Q^{2}\right)}},
$$

where $\lambda\left(Q^{2}\right)$ is the power of the fit of inclusive data to the form $F_{2}\left(x, Q^{2}\right) \sim x^{-\lambda\left(Q^{2}\right)}$ shown in Fig. 22 (left).

The expression in Eq. (4.3) leads to two important RENORM predictions:

- the Pomeron intercept in DDIS is the average of the soft quark and inclusive interceps,

$$
\alpha_{\mathbb{P}}^{D I S}=1+\lambda\left(Q^{2}\right), \quad \alpha_{\mathbb{P}}^{D D I S}=1+\frac{1}{2}\left[\varepsilon_{q}+\lambda\left(Q^{2}\right)\right] ;
$$

- the ratio of DDIS to DIS structure functions at fixed $\xi$ is independent of $x$ and $Q^{2}$,

$$
R\left[\frac{F_{2}^{D 3}\left(\xi, x, Q^{2}\right)}{F_{2}\left(x, Q^{2}\right)}\right]_{\xi}=\frac{1}{3} \cdot \frac{A_{\text {norm }}}{\xi^{1+\varepsilon}} .
$$

Data from ZEUS and $\mathrm{H} 1$ are consistent with these predictions [19].

At low $Q^{2}$, the gap probability saturates and renormalization must be applied [15]. In the RENORM model, the resulting suppression factor depends on the size of the rapidity interval available for particle production, which is $\Delta y^{\prime}=\ln s-\ln Q^{2}=\ln \left(s / Q^{2}\right)$. For dijet photoproduction, where $\Delta y^{\prime} \approx 6$, a suppression factor of $\approx 3$ is expected by RENORM [15], similar to that observed in soft SD at the Tevatron. Data from dijet photoproduction at HERA are consistent with this prediction [6, 7]. Moreover, since the suppression in RENORM is due to saturation of the gap probability, the same suppression is expected for both direct and resolved rates at low $Q^{2}$ DDIS. This prediction is also consistent with observation [6, 7]. 
At the Tevatron, where the gap probability saturates and must be renormalized to unity, the RENORM diffractive structure function takes the form

$$
\begin{array}{r}
F_{J J}^{D 3}\left(\xi, x, Q^{2}\right)=N_{\text {gap }}^{-1}(s, \beta) \cdot \frac{1}{\xi 1+2 \varepsilon} \cdot F_{J J}\left(\frac{x}{\xi}, Q^{2}\right) \\
N_{\text {gap }}(s, \beta)=\int_{\xi_{\text {mim }}}^{\xi=0.1} \frac{d \xi}{\xi^{1+2 \varepsilon}} \approx \frac{(\beta s)^{2 \varepsilon}}{2 \varepsilon},
\end{array}
$$

where, as in DDIS, $\varepsilon$ is the power from the fit of the soft structure function to the form $x f(x) \sim x^{-\varepsilon}$, and the limits $\xi_{\text {min }}=x_{\min } / \beta$ and $x_{\min }=1 / s$ are used [15]. Through renormalization, $F_{J J}^{D 3}$ acquires a factor $\sim(1 / \beta)^{2 \varepsilon}$, and the diffractive to inclusive structure function ratio, $R_{J J}(S D / N D)$, a factor $\sim(1 / x)^{2 \varepsilon}$. Since from color factor considerations $F_{J J}$ is gluon dominated,

$$
F_{J J}(x)=x\left[g(x)+\frac{4}{9} q(x)\right],
$$

where $g(x)$ and $q(x)$ are the gluon and quark densities in the proton, the relevant parameter $\varepsilon$ is the parameter $\lambda_{g}$ of Fig. 目 (right), resulting in $R_{J J}(S D / N D) \sim 1 / x^{0.4}$. This RENORM prediction is confirmed by the CDF data, where the $x$-dependence of the diffractive to inclusive ratio was measured to be $\sim 1 / x^{0.45 \pm 0.02}$ [21].

\section{Conclusion}

A phenomenological model has been presented (RENORM), in which diffractive cross sections are obtained from parton-level cross sections and the underlying inclusive parton distribution function of the interacting hadrons using QCD color factors and appropriate (re)normalization. Scaling and factorization properties observed in fixed target, Tevatron, and HERA data form both the basis and a testing ground for RENORM.

In soft diffraction, normalized single-gap and double-gap differential cross sections (SD, DD, DPE, SDD) are obtained in RENORM in terms of two free parameters, $\varepsilon$ and $\alpha^{\prime}$, which are identified as those of the Regge theory soft Pomeron trajectory, $\alpha(t)=1+\varepsilon+\alpha^{\prime} t$. Furthermore, the $\varepsilon$ is obtained from the color-factor weighted exponents of power law fits to soft nucleon PDFs, leaving $\alpha^{\prime}$ as the only free parameter in the model.

In hard diffraction, RENORM is applied to HERA and Tevatron data. At HERA, interesting features of the data include: $\varepsilon\left(Q^{2}\right)_{D D I S}<\varepsilon\left(Q^{2}\right)_{D I S}, F_{2}^{D 3}\left(\xi, x, Q^{2}\right) /\left.F_{2}\left(x, Q^{2}\right)\right|_{\xi} \sim$ constant, dijet photoproduction suppressed by factor of $\sim 3$, and direct / resolved low $Q^{2}$ DIS both suppressed by approximately the same factor relative to high $Q^{2}$ DIS. At the Tevatron, features of the data include: cross sections at fixed collision energy are flavor independent, the ratio of SD/ND rates decreases with increasing $x_{B j}$, and the DSF of the proton in DPE events with a leading ${ }^{-} p$ is suppressed relative to that in SD. Comparing HERA with Tevatron results, factorization breaks down at the Tevetron relative to HERA by a factor of $\sim \mathscr{O}(10)$, but is restored in comparing the double-gap DSF obtained from DPE at CDF with that obtained from DDIS of HERA. All these features are successfully interpreted by RENORM.

In conclusion, in light of the success of RENORM in describing the data, diffraction may be viewed as an interaction between low- $x$ partons subject to color constraints. 


\section{References}

[1] J.D. Bjorken, Phys. Rev. D 47, 101 (1993).

[2] P. D. B. Collins, An Introduction to Regge Theory and High Energy Physics, Cambridge University Press (1977); V. Barone and E. Predazzi, High-Energy Particle Diffraction, Springer Press (2001); S. Donnachie, G. Dosch, O. Nachtmann, and P. Landshoff, Pomeron Physics and QCD, Cambridge University Press (2002).

[3] K. Goulianos, Hadronic Diffraction: Where do we Stand?, in La Thuile 2004, Results and Perspectives in Particle Physics, edited by M. Greco, Proc. of Les Rencontres de Physique de la Vallé d'Aoste, La Thuile, Aosta Valley, Italy, February 29 - March 6, 2004, pp. 251-274; arXiv:hep-ph/0407035.

[4] K. Goulianos and J. Montanha, Phys. Rev. D 59, 114017 (1999).

[5] Konstantin Goulianos, Diffraction at the Tevatron: CDF Results, in these Proceedings

[6] B. Loehr, Inclusive Diffraction at HERA from the ZEUS Experiment, in these Proceedings.

[7] P. Laycock, Inclusive Diffraction in DIS - HI Results, in these Proceedings.

[8] F. Abe et al. (CDF Collaboration), Phys. Rev. D 50, 5518 (1994).

[9] F. Abe et al. (CDF Collaboration), Phys. Rev. D 50, 5535 (1994).

[10] F. Abe et al. (CDF Collaboration), Phys. Rev. D 50, 5550 (1994).

[11] T. Affolder et al. (CDF Collaboration), Phys. Rev Lett. 87, 141802 (2001).

[12] D. Acosta et al. (CDF Collaboration), Phys. Rev. Lett. 93, 141601 (2004).

[13] D. Acosta et al. (CDF Collaboration), Phys. Rev Lett. 91, 011802 (2003).

[14] K. Goulianos, Phys. Reports 101, 171 (1983).

[15] K. Goulianos, Phys. Lett. B 358, 379 (1995); Erratum-ib. 363, 268 (1995).

[16] K. Goulianos, in Diffraction in QCD, Corfu Summer Institute on Elementary Particle Physics, Corfu, Greece, 31 Aug - 20 Sep 2001; e-print Archive: hep-ph/0203141.

[17] E. Levin, in An Introduction to Pomerons, Preprint DESY 98-120.

[18] R.J.M. Covolan, J. Montanha, and K. Goulianos, Phys. Lett. B 389, 176, (1996).

[19] F.P. Schilling (H1 Collaboration), in Measurement and NLO DGLAP QCD Interpretation of Diffractive Deep-Inelastic Scattering at HERA, submitted to $31^{\text {st }}$ International Conference on High Energy Physics, ICHEP02, Amsterdam, The Netherlands, Jul. 24-31, 2001 (abstract 980).

[20] J. Collins, J. Phys. G 28, 1069 (2002); arXiv:hep-ph/0107252.

[21] T. Affolder et al. (CDF Collaboration), Phys. Rev. Lett. 84, 5043 (2000). 